\title{
Chronic Takotsubo Cardiomyopathy after Coronary Bypass Surgery Caused by Hypothyroidism on Levothyroxine Replacement Therapy
}

\author{
Stefan Peters \\ St. Elisabeth Hospital gGmbH Salzgitter, Germany \\ ${ }^{\star}$ Correspondence to: Prof. Dr. med.Stefan Peters, Cardiology, St. Elisabeth Hospital gGmbH Salzgitter, Liebenhaller Str. 20, 38259 Salzgitter, Germany; \\ E-Mail: H.u.S.Peters@t-online.de
}

Received: February 27, 2018; Accepted: March 31, 2018; Published: April 06, 2018;

\section{Case Report}

A meanwhile 81-year old female patient was recurrently admitted to hospital because of dyspnoea and bilateral edema.

13 years ago (in 2005) the patient had coronary bypass surgery with normally contracting left ventricle with an ejection fraction of $64 \%$.

She had severe hypothyroidism and was on levothyroxine replacement therapy.

In 2014 the patient developed atrioventricular node tachycardia and was treated with beta blocking agents and amiodarone. In the same year she developed high-grade atrioventricular block and DDD pacemaker was implanted.

At the end of 2014 echocardiography revealed asynchronous septal left ventricular hyokinesia due to continuous right ventricular stimulation with an ejection fraction of $58 \%$.

In the next hospital stay in february 2015 the patient presented with massive dyspnoea and massive bilateral edema. Echocardiography revealed reduction of left ventricular function with an ejection fraction of $40 \%$, and apical ballooning. Because of the echocardiographic finding coronary angiography was initiated and showed normal bypass morphology without any stenoses. She was still on levothyroxine replacement therapy at a dosage of $125 \mathrm{ug}$ oral medication.

In the next years she was recurrently hospitalised with biventricular heart failure and echocardiographic reduction of left ventricular function, and continuous apical ballooning.

In conclusion, in the first view DDD pacemaker implantation seemed to contribute to reduction of echocardiographic left ventricular function but is limited to asynchronous septal hyokinesia. In a second view chronic takotsubo cardiomyopathy despite well functioning bypass grafting caused by severe hypothyroidism on current levothyroxine replacement therapy was debated.

Takotsubo cardiomyopathy caused by pheochromocytome [1] or hyperthyroidism [2] is well known due to cathecholamine increase.
Severe hypothyroidism seems to be a reason for takotsubo cardiomyopathy $[3,4]$ or on levothyroxine replacement therapy as well [5]. Due to ongoing hypothyroidism takotsubo cardiomyopathy is in a chronic state and left ventricular recovery is not yet reported during a long period of time from 2015 until 2018.

The theory is that reversible or continuous left ventricular dysfunction is caused by intense, neuroadrenergic myocardial stimulation, as it appears in severe hypothyroidism although on levothyroxine replacement therapy. This phenomenon appears to be the main trigger despite multiple described mechanisms.

\section{References}

1. Gagnon N, Mansour S, Bitton Y, Bourdeau I (2017) Takotsubo-like cardiomyopathy in a large cohort of patients with pheochromocytoma and paraganglioma. Endocr Pract. 23: 1178 - 1192

2. Miyazaki S, Kamiishi T, Hosokawa N, Komura M, Sagai H, Takamoto T (2004) Reversible left ventricular dysfunction takotsubo cardiomyopathy associated with hyperthyreoidism. Jpn Heart J 45: 889-94

3. Aggarwal S, Papani R, Gupta V. Can thyroid break your heart? (2015) Role of thyroid in takotsuvo cardiomyopathy: A single center retrospective study. In $J$ Cardiol 184: 545-6

4. Micallef T, Gruppetta M, Cassar A, Fava S (2011) Takotsubo cardiomyopathy and severe hypothyroidism. J Cardiovasc Med (Hagerstown) 12: 824-827. [crossref]

5. Madias JE (2015) Is hypothyroidism (on levothyroxine replacement) a precipitant of Takotsubo syndrome? Int J Cardiol 187: 29-30. [crossref]

Citation:

Stefan Peters (2018) Chronic Takotsubo Cardiomyopathy after Coronary Bypass Surgery Caused by Hypothyroidism on Levothyroxine Replacement Therapy. Cardiac Sci Res $J$ Volume 1(3): 1-1 